\title{
A New Approach to the Particle-in-Cell Method for the Computation of Three-Dimensional Incompressible Unsteady Flows
}

\author{
F. Buron, L. Pérault \\ Laboratoire d'Etudes Aérodynamiques \\ de l'université de Poitiers \\ UMR CNRS 6609 \\ Ecole Nationale Supérieure de Mécanique et d'Aérotechnique \\ 86960 Futuroscope Cedex, France \\ (buron@lea.ensma.fr/perault@lea.ensma.fr)
}

\begin{abstract}
A hybrid three-dimensional particle-in-cell (PIC) method is proposed. This 3D PIC technique combined with a classical three-dimensional vortex particle method is applied to the calculation of unsteady inviscid and incompressible flow. More precisely the wake roll-up behind an inclined flat plate is studied. A spreading procedure of the vorticity, a direct interaction between nodes of the grid and a mesh generated from the plate discretization present a few undesirable grid effects, but ensure a continuous application of the slip condition to the plate. In this way the total circulation is nearly conserved and, in the case of a separated flow at high angle of attack, the particles are prevented from crossing the wing. Numerical results are compared with experiment for several angles of attack and aspect ratios.
\end{abstract}

\section{Introduction}

In this paper we describe a new approach of the particle-in-cell (PIC) method for the computation of three-dimensional incompressible flows. The simulation of the wake roll-up behind a flat plate, in the case of an inviscid flow, is considered for several aspect ratios and different angles of attack. Vortex-lattice and panel methods are often used in preliminary design $[1,2,3]$ but these methods present some limits in the simulation of strong wake roll-up giving a poor resolution of the wake. In the case of an inviscid flow, vortex filament methods $[4,5]$ or vortex particle methods (VPM) [6] would be a more appropriate design tool to provide an accurate description of the flow in vorticity confinement regions. Nevertheless these methods present some undesirable aspects. First a large number of particles are required for reasonable accuracy and then Biot-Savart interactions between all the vortex elements contained in the computational domain induce an excessive computation time, which scales as the square of the number of vortices. On the other hand when vortex elements are much too close to each other, the singular character of the method brings about some high induced velocities or high distortion rates of the vortex vector in the calculation of the stretching. Even using robust regularization functions the computation is troubled by numerical instabilities. The motion of the vortices and the stretching step are less accurate. In the case of wake-body interactions, when particles are near the panels of the plate and without any numerical device, the vortex sheet presents some instabilities which quickly cause the calculation to breakdown. For a separated flow, in the leading edge area, some particles may even cross the wing. The present work applies a hybrid PIC method to the wing wake roll-up problem and attempts to use the stabilizing nature and the quickness of the «cell» method to speed up the calculations and, with an Eulerian grid generated from the plate, to prevent the particles from crossing it. 


\section{Numerical model}

\subsection{Basic principles}

For an inviscid 3D flow the equation of the motion is given by :

$$
\frac{\partial \mathbf{u}}{\partial \mathrm{t}}+(\mathbf{u} \cdot \nabla) \mathbf{u}=-\frac{1}{\rho} \nabla \mathrm{p}
$$

where $p, \rho$ are respectively the pressure and the density, and $\mathbf{u}(x, t)$ the velocity field. In the vortex particle method we want to trace the motion of the vortices according to a classical Lagrangian vortex tracking concept. All the particles form the vorticity field,

$$
\omega=\nabla \times \mathbf{u},
$$

and the vorticity transport equation (Helmholtz equation) is obtained by taking the curl of (1):

$$
\frac{\mathrm{D} \omega}{\mathrm{Dt}}=\frac{\partial \omega}{\partial \mathrm{t}}+(\mathbf{u} \cdot \nabla) \omega=(\omega \cdot \nabla) \mathbf{u}
$$

using the equation of continuity (incompressibility condition),

$$
\nabla \cdot \mathbf{u}=0
$$

A position vector $\mathbf{X}$ and a strength vector $\Omega$ (volume $\times$ vorticity) are associated to each vortex element and the particle representation of the vorticity field is taken as :

$$
\widetilde{\omega}_{\sigma}(x, t)=\sum_{p=1}^{N p(t)} \Omega_{p}\left(X_{p}, t\right) \zeta_{\sigma}\left(\left|x-X_{p}\right|\right),
$$

where $\zeta_{\sigma}$ is a regularization function and $\sigma$ the core size (cut off length), i.e.,

$$
\zeta_{\sigma}(\mathrm{x})=\frac{1}{\sigma^{3}} \zeta\left(\frac{|\mathrm{x}|}{\sigma}\right)
$$

In our case we have chosen the high order algebraic smoothing proposed by Winckelmans and Leonard in [6] for its convergence properties and its greater numerical convenience. It is written as :

$$
\zeta(\rho)=\frac{15}{8 \pi} \frac{1}{\left(\rho^{2}+1\right)^{\frac{7}{2}}}
$$

The particles are convected by the local velocity and the strength vector is trained by the local velocity gradient according to the following two step integro-differential scheme with time :

$$
\left\{\begin{array}{l}
\frac{\mathrm{D} \mathbf{X}_{\mathrm{p}}}{\mathrm{Dt}}=\mathbf{U}_{\mathrm{p}}\left(\mathbf{X}_{\mathrm{p}}, \mathrm{t}\right) \\
\frac{\mathrm{D} \boldsymbol{\Omega}_{\mathrm{p}}}{\mathrm{Dt}}=\left(\boldsymbol{\Omega}_{\mathrm{p}} \cdot \nabla\right) \mathbf{U}_{\mathrm{p}}\left(\mathbf{X}_{\mathrm{p}}, \mathrm{t}\right)
\end{array}\right.
$$


Alternative forms of the vorticity equation (3) can be written as a mixed scheme for the deformation tensor (used by Rehbach [7]) or a transpose scheme :

$$
\frac{\mathrm{D} \omega}{\mathrm{Dt}}=(\omega \cdot \nabla)^{\mathrm{T}} \mathbf{u}
$$

We use this transpose scheme because it leads to the exact conservation of the total vorticity [8] and leads to a weak solution of equation (9) as shown by [9]. These conditions are not satisfied by the classical scheme (3). The velocity field is obtained as :

$$
\mathbf{U}_{\sigma}(\mathrm{x}, \mathrm{t})=\sum_{\mathrm{p}=1}^{\mathrm{Np}(\mathrm{t})} \mathbf{K}_{\sigma}\left(\mathrm{x}-\mathbf{X}_{\mathrm{p}}\right) \times \boldsymbol{\Omega}_{\mathrm{p}}\left(\mathbf{X}_{\mathrm{p}}, \mathrm{t}\right)
$$

where $\mathbf{K}_{\sigma}(\mathrm{x})$ is the regularized Biot-Savart kernel.

\subsection{Emission scheme}

For the plate discretization we use the classical boundary element method and according to the lifting surface theory, the wing is modeled by a repartition of panels with attached surface normal doublets. Writing the continuity of the pressure through the vortex sheet and applying Bernoulli's equation to both sides of the emission line we obtain :

$$
\frac{\partial \mu}{\partial \mathrm{t}}+\mathbf{U}_{\mathrm{m}} \cdot \nabla \mu=\frac{\mathrm{D} \mu}{\mathrm{Dt}}=0
$$

with $\mathbf{U}_{\mathrm{m}}=\frac{1}{2}\left(\mathbf{U}_{+}+\mathbf{U}_{-}\right),+$for the upper surface, - for the lower surface. This equation (11) means that the doublet strenght $\mu_{\mathrm{p}}$ of each panel does not vary with time. Thus the vortices, at each time step, are obtained, in a continuous way, shedding virtual panels symmetrically to the emission line. Subsequently they are converted into particles and their intensity vectors are taken as :

$$
\Omega_{\mathrm{p}}=\iint_{\mathrm{Si}}\left(\mathbf{n}_{\mathrm{p}} \times \nabla_{\mathrm{p}} \mu_{\mathrm{p}}\right) \mathrm{ds},
$$

the $\mu_{\mathrm{p}}$ being determined by the slip condition satisfied on each panel of the plate. This volume discretization of the continuous vorticity field was developed in 1977 by Rehbach [7] and several extensions of the method have been made around bodies in arbitrary motion (rotors or propellers, freesurface flow with lifting effects), see [10,11, 12] for more details.

\section{Particle-in-Cell technique}

The PIC method is based on a simple technique. During the calculation we follow the vortices in a Lagrangian frame but an Eulerian grid is used to compute the velocity field required to move the particles. Historically this method has its origin in two-dimensional computations [13, 14, 15]. This method was applied in 3D simulations using vortex filaments $[16,17]$. Our approach differs from the classical vortex-in-cell (VIC) method in that, rather than generate a grid stream function using the stream function-vorticity finite-difference technique, we kept a direct induction law (Biot-Savart) between nodes of the grid. 


\subsection{Mesh generation}

To obtain the grid vorticity we use an adaptive volume mesh, adaptive in the fact that it is directly generated from the flat plate discretization. The mesh is made up of triangular section cells. They are more appropriate than quadrangular section cells to create an accurate mesh around an arbitrary profile. Only 6 nodes instead of 8 for a quandrangular cell, need to be taken into account. The plate is modeled by a regular distribution of panel strips in the $y$ direction (see Fig. 1). The triangular sections of the volume cells are located in the $y$ position of the panel control points. The nodes of the first cells around the body are the control points of the plate whatever its shape.

\subsection{Spreading function and interpolation scheme}

In the first part a grid vorticity is generated by distributing the vorticity from each particle over the six neighboring grid points of a cell (Fig. 2). The spreading procedure is based on a volume weighting law (13). This scheme provides local conservation of total vorticity and weak undesirable grid effects. The $\mathrm{P}_{\mathrm{i}}$ are the weights of the spreading procedure. For a vortex, a cell is detected and then the $P_{i}$ are easily obtained by dividing each volume $V_{i}$, opposed to the $i$ node, by the total volume $\mathrm{V}_{\mathrm{T}}$ of the cell. In term of Long-Range Constraint (14) [18], the zeroth-order constraint and the first-order constraint are satisfied.

$$
\begin{aligned}
& \left\{\begin{array}{l}
\sum_{\mathrm{i}=1}^{6} \Omega_{\mathrm{i}}=\sum_{\mathrm{i}=1}^{6} \mathrm{P}_{\mathrm{i}} \Omega_{\mathrm{p}}=\Omega_{\mathrm{p}} \\
\mathrm{P}_{\mathrm{i}}=\frac{\mathrm{V}_{\mathrm{i}}}{\mathrm{V}_{\mathrm{T}}}, \quad \sum_{\mathrm{i}=1}^{6} \mathrm{P}_{\mathrm{i}}=1
\end{array}\right. \\
& \sum_{\mathrm{p}=1}^{6} \mathrm{P}_{\mathrm{i}} \Delta_{\mathrm{i}}^{\mathrm{n}}=0, \quad \text { with } \Delta_{\mathrm{i}}^{\mathrm{n}}=\left(\mathrm{x}-\mathrm{x}_{\mathrm{i}}\right)^{\mathrm{n}},
\end{aligned}
$$

For each particle the fraction of the vorticity vector is added to the previous contributions of the vortices distributed on the i node. Here the method differs from a classical VIC method. We obtain the grid velocity using a direct induction law (Biot-Savart) between nodes of the grid. In this way we compute the velocity and the velocity gradient tensor only on mesh points supporting vorticity. Finally, by the same numerical filter (15) we interpolate velocity and gradient values from the grid to the particles and we get new position and new vortex vector by solving (8).

$$
\left\{\begin{array}{l}
\mathbf{U}_{\mathrm{p}}=\sum_{\mathrm{i}=1}^{6} \mathrm{P}_{\mathrm{i}} \mathbf{U}_{\mathrm{i}} \\
\nabla \mathbf{U}_{\mathrm{p}}=\sum_{\mathrm{i}=1}^{6} \nabla \mathbf{U}_{\mathrm{i}}
\end{array}\right.
$$

We resolve the both equations simultaneously and without interaction between them. This direct scheme (Fig. 3) requires 3 projection-interpolation operations instead of 4 for a splitting scheme.

\subsection{Advantages of the method}


The application of the present technique brings some advantages to the fore. Firstly, the PIC method is computationnally more efficient, resulting in the reduction of the CPU time. For the classical VPM, the number of operations for each time step is of the order $\mathrm{O}\left(\mathrm{P}^{2}\right)$ where $\mathrm{P}$ is the number of particles. However, for the current procedure the number of operations is reduced to $\mathrm{O}\left(\mathrm{N}^{2}\right)$ for each time step where $\mathrm{N}$ is only the number of nodes supporting vorticity. Obviously this gain increases with the number of vortices.

Secondly, the velocities and gradients are always computed on fixed nodes. This technique removes infinite values and ensures a more regular wake motion as well as a good conservation of the vorticity field during transport equation treatment. Using Biot-Savart interactions between grid points this method reduces numerical diffusion and prevents the calculation of the streamfunction which is not generally divergence-free. Admittedly, unlike for the vortex filament methods, the particle field is not generally divergence-free. Initially $\widetilde{\omega}_{\sigma}(x, t)$ can be set to be a good representation of $\omega(x, t)$, but the classical method has no built-in control on keeping the particle vorticity field nearly divergencefree as time evolves. For example when two intensity vectors are perpendicular they could, during their interactions, induce unusually strong deformation rates of the vortex vector. This weakness may get the method into trouble in long time computations. The present PIC technique reduces the number of strong deformations of $\Omega_{p}$ and preserves a better conservation of the total vorticity.

Finally, the adaptive volume mesh is a good tool to avoid strong interactions between vortex elements, especially to eleminate strong wake-body interactions which appear in the case of a separated flow at high angle of attack when particles of leading edge come across the flat plate. Indeed, the grid nodes located on the body and supporting vorticity are always panel control points (see Fig. 1). In this way they received special treatment. On one hand the normal doublet surface distribution induces in the center of the panel a tangential velocity gradient [19] which is necessary to be added to the total velocity on the control point :

$$
\begin{cases}\mathbf{U}_{\text {iplate }}=\mathbf{U}_{\text {iplate }}-\frac{\nabla \mu}{2} & \text { for upper surface } \\ \mathbf{U}_{\text {iplate }}=\mathbf{U}_{\text {iplate }}+\frac{\nabla \mu}{2} & \text { for lower surface }\end{cases}
$$

On the other hand we compute on these special nodes the total velocity with the classical VPM to be sure to annul the normal velocity component. This procedure, intrinsic to the present PIC technique, insures a more homogeneous repartition of the slip condition and prevents the particles from crossing the plate.

At the moment, no particle addition and no relaxation of $\nabla \widetilde{\omega}_{\sigma}[6]$ was used to maintain a divergence-free particle vorticity field.

\section{Results}

Firstly, this process is applied to the attached flow around an $A R=1$ flat plate. Due to the symmetrical nature of the flow we take into account only half of the study domain. The vortices are shed from the trailing edge and the side-edge of the plate. The half plate is modeled by $50(10 \times 5)$ panels. Figure 5 shows the number of strong deformation rates of vortex vectors higher than $20 \%$ at $\alpha=7.4$ degrees. We note that infinite values in the computation of the stretching are more numerous for the classical VPM. On the contrary, the present PIC method reduces the case of unrealistically high interactions between intensity vectors and the maximum of distortion rates higher than $20 \%$ is equal to 1 . This ensures a better conservation of the wake circulation during transport equation treatment. Figure 7 shows the particle vorticity field of an $\mathrm{AR}=1$ flat plate at $\alpha=11.3$ degrees. We observe the 
intensity vectors of the particles at $\mathrm{t}^{*}=2\left(\mathrm{t}^{*}=\mathrm{t} \mathbf{U}_{\infty} / \mathrm{c}:\right.$ non-dimensional time, $\mathbf{U}_{\infty}:$ upstream velocity, $\mathrm{c}$ : unit chord). Notice that the present computations used a much larger number of vortices. There were $20 \times 10$ panels in the half wing, that is to say 2400 particles at the final time step. We can see that the present PIC method results in an increase in structured vectors so that we obtained better vortex lines patterns (Fig. 7(b)). Figure 6 shows the conservation of the circulation obtained from the first numerical application. The stabilizing nature of the method ensures a more realistic evolution of the wake circulation and so the total circulation is nearly conserved.

Figure 8 presents the normal force coefficients obtained for an $A R=1$ flat plate at $t^{*}=5(1500$ particles) for several angles of attack. The case $\alpha=1.6$ degrees is computed with no side-edge wake. The results are in a good agreement with experiment [20,21], particularly for the steady values given by Scholz. Normal force coefficients are also obtained for an $A R=2$ plate (Fig. 9). We notice that our results are close to the experiment [20] but beginning at about $\alpha=12$ degrees the results start to deviate from the experimental data as the leading edge wake has not been computed. Also with respect to the physical case, this is the angle of attack range where the unsteady stall appears.

Wake roll-up of a separated flow, for an $\mathrm{AR}=1$ flat plate at $\alpha=25$ degrees, is shown in figure 10. In the case of the classical VPM (Fig. 10(a)), without special treatment or numerical device, during wake-body interactions some particles cross the plate and the sheet is rapidly broken. The application of the present PIC process (Fig. 10(b)) prevents those leading edge vortex sheet instabilities. Figure 4 brings the speed of the method to the fore. For an $A R=1$ flat plate wake simulation the gain in computational time is about 3.6 for 1680 particles. The computing time for the classical VPM was about $19800 \mathrm{CPU}$ seconds to reach 112 time steps ( $(*=5.6)$ instead of about $5600 \mathrm{CPU}$ seconds for the present method. The calculations were carried out on 2 processors of a Modulux PC computer. For unsteady separated flows the PIC method should presents even greater time savings.

\section{Conclusion}

The combination between the classical 3D VPM and this hybrid 3D PIC method gives encouraging results. The accurate description of the wake roll-up given by the basic VPM is improved by the stabilizing nature of the PIC technique. On one hand, with the spreading function of the vorticity and the direct interactions between nodes of the mesh, the method presents few undesirable grid effects and avoids strong distortion rates of intensity vectors, nearly conserving total circulation. On the other hand, with a mesh generated from the body discretization, the slip condition is verified over the whole plate making it impermeable. In this way leading edge numerical instabilities are eliminated. Finally the PIC implementation provides faster calculations. We hope that these advantages will soon allow us to compute flows around more complex bodies like NACA airfoils or delta wings.

\section{References}

[1] Maskew, B., «Predicting Aerodynamic Characteristics of Vortical Flows on Three-Dimensional Configurations using a Surface-Singularity Panel Method,» AGARD CP-342, 1983

[2] Katz, J. and Plotkin, A., Low-Speed Aerodynamics - From Wing Theory to Panel Methods, McGraw-Hill, New York, 1991

[3] Johnson, F. T., Tinoco, E. N., Lu, P. and Epton, M. A., «Three-Dimensional Flow over Wings with Leading-Edge Vortex Separation,» AIAA Journal, 14, 367-380, 1980

[4] Leonard, A., «Computing Three-Dimensional Incompressible Flows with Vortex Elements,» Ann. Rev. Fluid. Mech., 17, 523-559, 1985 
[5] Knio, O. M. and Ghoniem, A. F., «Numerical Study of a Three-Dimensional Vortex Method,» $J$. Comp. Phys., 86, 75-106, 1990

[6] Winckelmans, G. S. and Leonard, A., «Contributions to Vortex Particle Methods for the Computation of Three-Dimensional Incompressible Unsteady Flows,» J. Comp. Phys., 109, 247-273, 1993

[7] Rehbach, C., «Calcul numérique d'écoulements tridimensionnels instationnaires avec nappes tourbillonnaires,» La Rech. Aérosp., 5, 289-298, 1977

[8] Choquin, J-P. and Cottet, G-H., «Sur l'analyse d'une classe de méthodes de vortex tridimensionnelles,» C. R. Acad. Sci., 306, 739-742, 1988

[9] Winckelmans, G. S. and Leonard, A., «Weak Solutions of the Three-Dimensional Vorticity Equation with Vortex Singularities,» Phys. Fluids., 31, 1988

[10] Cantaloube, B. and Huberson, S., «Calcul d'écoulements de fluide incompressible non visqueux autour de voilures tournantes par une méthode particulaire,» La Rech. Aérosp., 6, 403-415, 1984

[11] Billet, G., «Simulation numérique d'un décollement tridimensionnel pariétal,» La Rech. Aérosp., 4, 229-240, 1980

[12] Rouffi, F., «Résolution numérique de problèmes non linéaires de l'hydrodynamique navale: manoeuvrabilité et tenue à la mer de navires,» Thèse de l'Université Pierre et Marie Curie, LIMSI, 1992

[13] Christiansen, J. P., «Numerical Simulation of Hydrodynamics by the Method of Point Vortices,» J. Comp. Phys., 13, 363-379, 1973

[14] Baker, G. R., «The Cloud-in-Cell Technique Applied to the Roll-up of Vortex Sheets,» J. Comp. Phys., 31, 76-95, 1979

[15] Pérault, L., «Calcul d'écoulements tourbillonnaires décollés par une méthode de convection cellulaire,» La Rech. Aérosp., 3, 63-71, 1993

[16] Couet, B., Buneman, O. and Leonard, A., «Simulation of Three-Dimensional Incompressible Flows with a Vortex-in-Cell Method,» J. Comp. Phys., 39, 305-328, 1981

[17] Ribeiro, R. S. and Kroo, I., «Vortex-in-Cell Analysis of Wing Wake Roll-Up,» Journal of Aircraft, 32, 962-969, 1995

[18] Hockney, R. W. and Eastwood, J. W., «Computer Simulation Using Particles», Institut of Physics Publishing, Adam Hilger, Bristol, 1988

[19] Bousquet, J., «Méthode des singularités», Cepadues éditions, Toulouse, 1990

[20] Scholz, N., «Kraft-und Druchverteilungsmessungen an Traflächen Kleiner Streckung,» Forscharb. Ing. Wes., 16, 85-91, 1949

[21] Winter, H., «Strömungsvorgänge an Platten und profilierten Körpern bei kleinen Spannweiten,» Forschg. Ing. Wes., 6, 67, 1935 


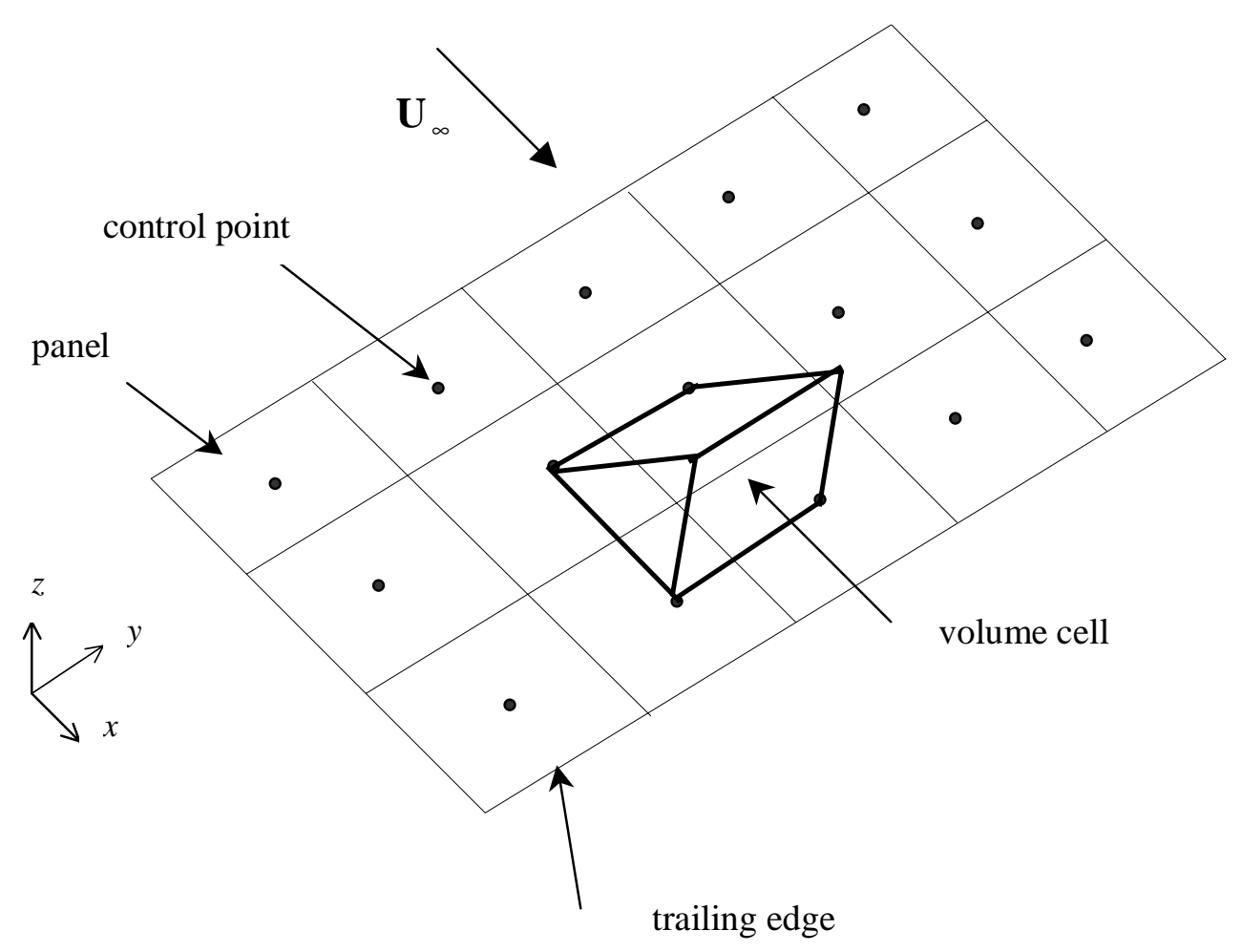

Figure 1: Adaptive mesh generation

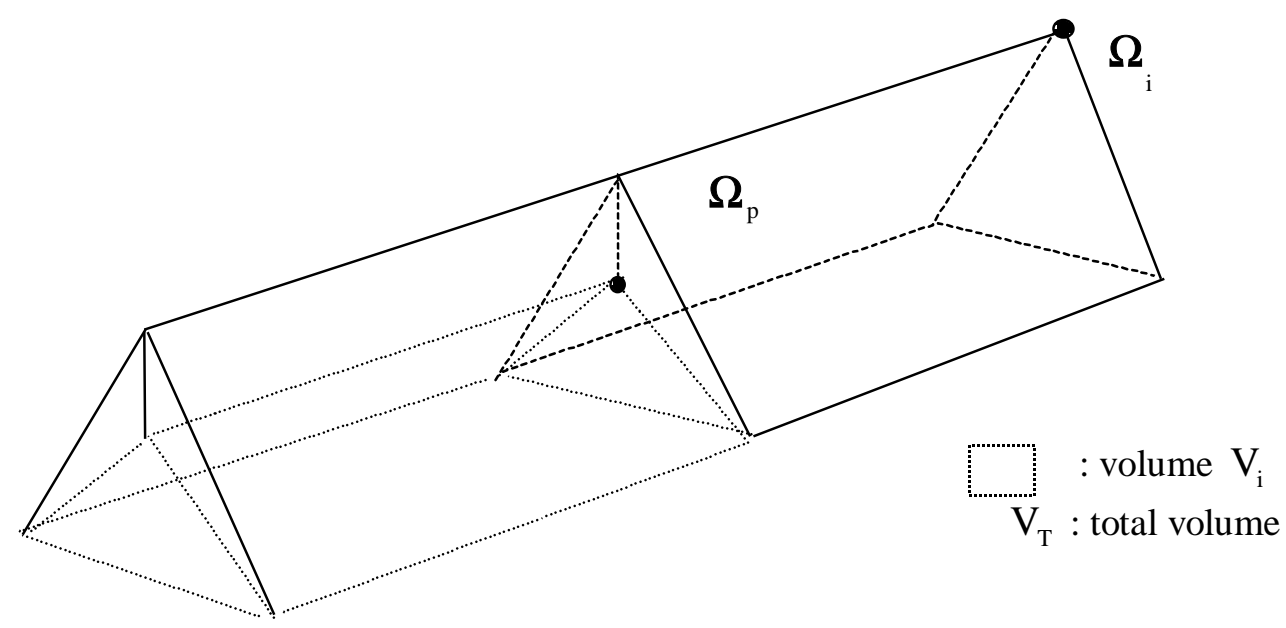

Figure 2: Triangular volume cell 

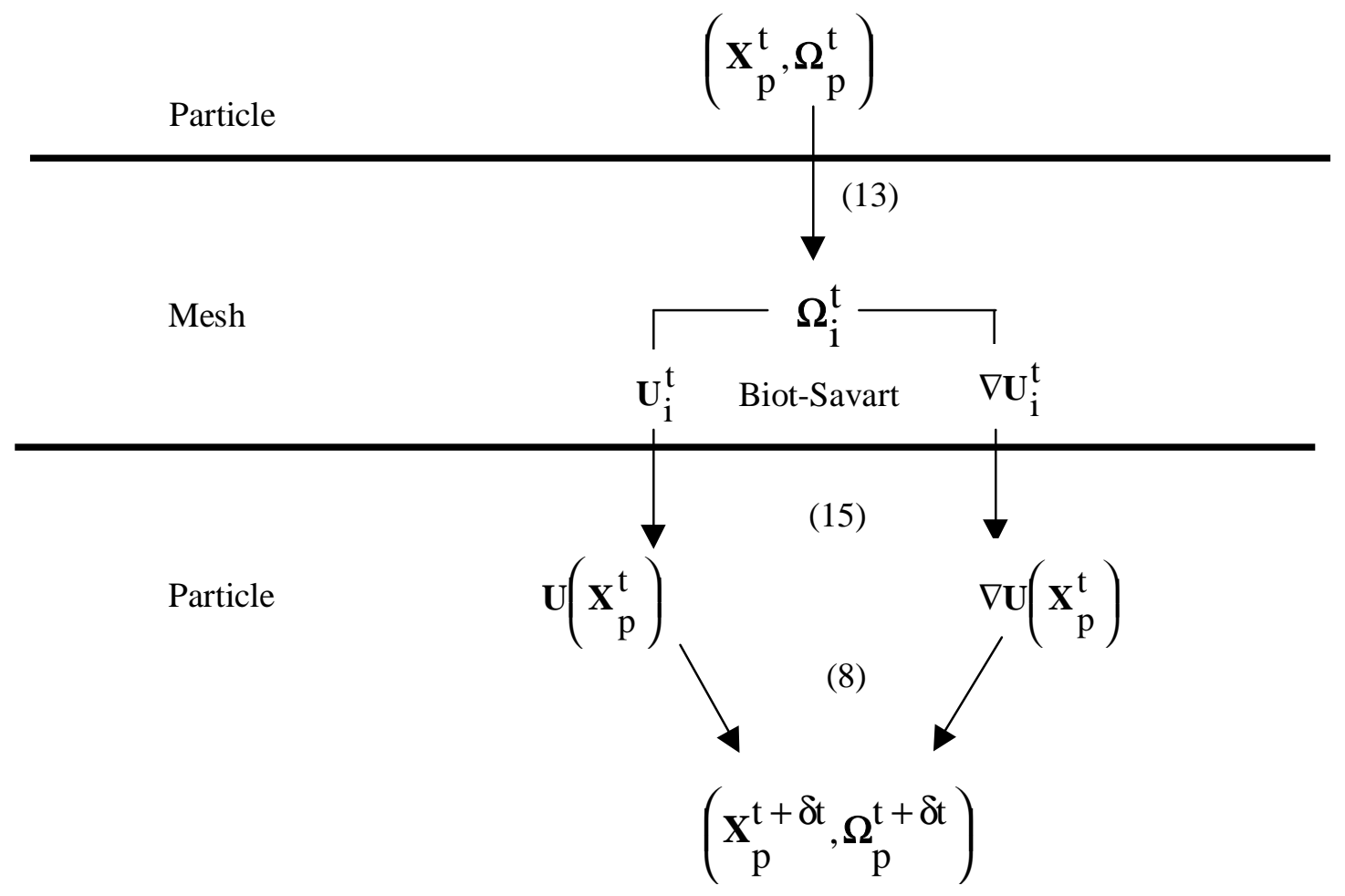

Figure 3: Direct scheme

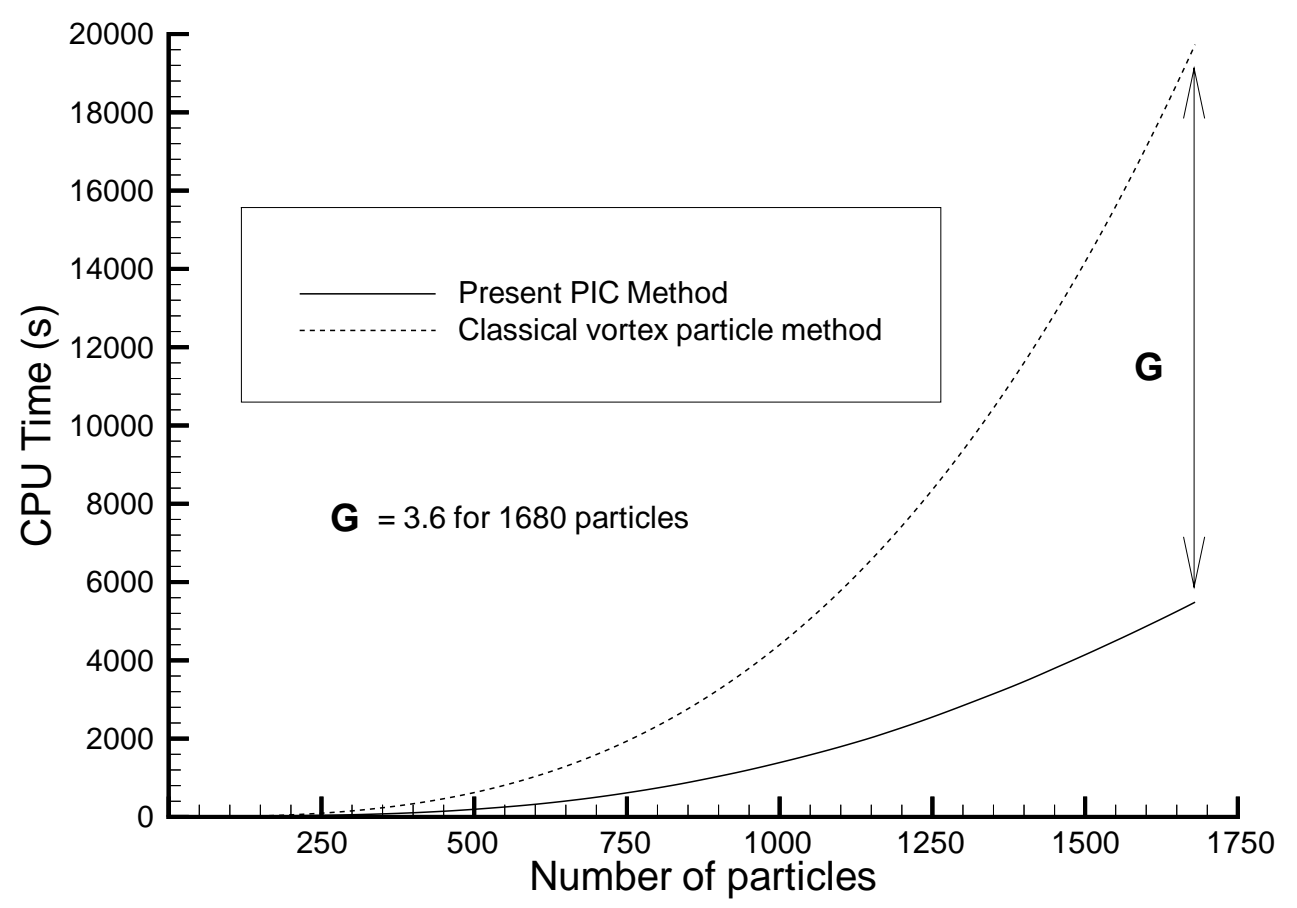

Figure 4: Computation time ( $\mathrm{AR}=1,15$ particles per time tep, 50 panels). 


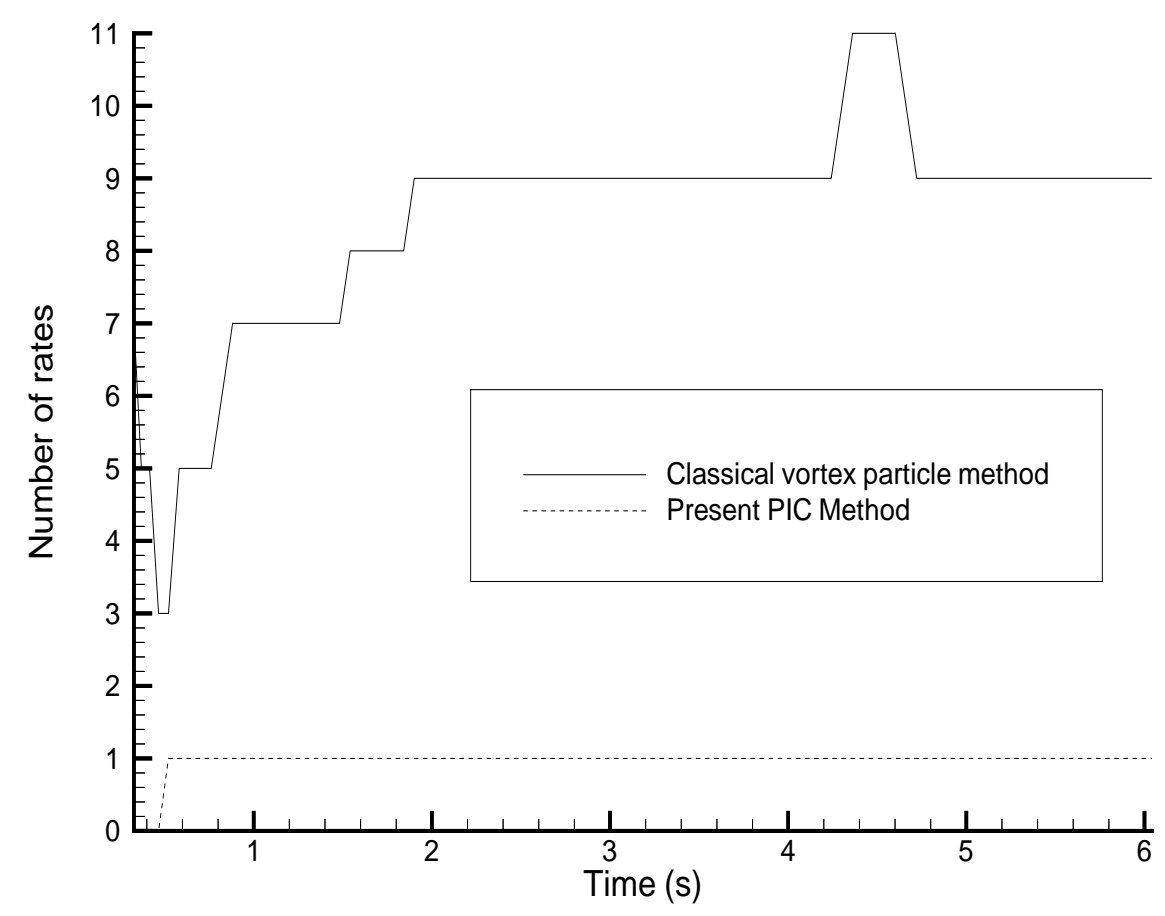

Figure 5: Number of distortion rates of vortex vectors higher than $20 \%$ ( $\mathrm{AR}=1, \alpha=7.4$ deg., 50 panels).

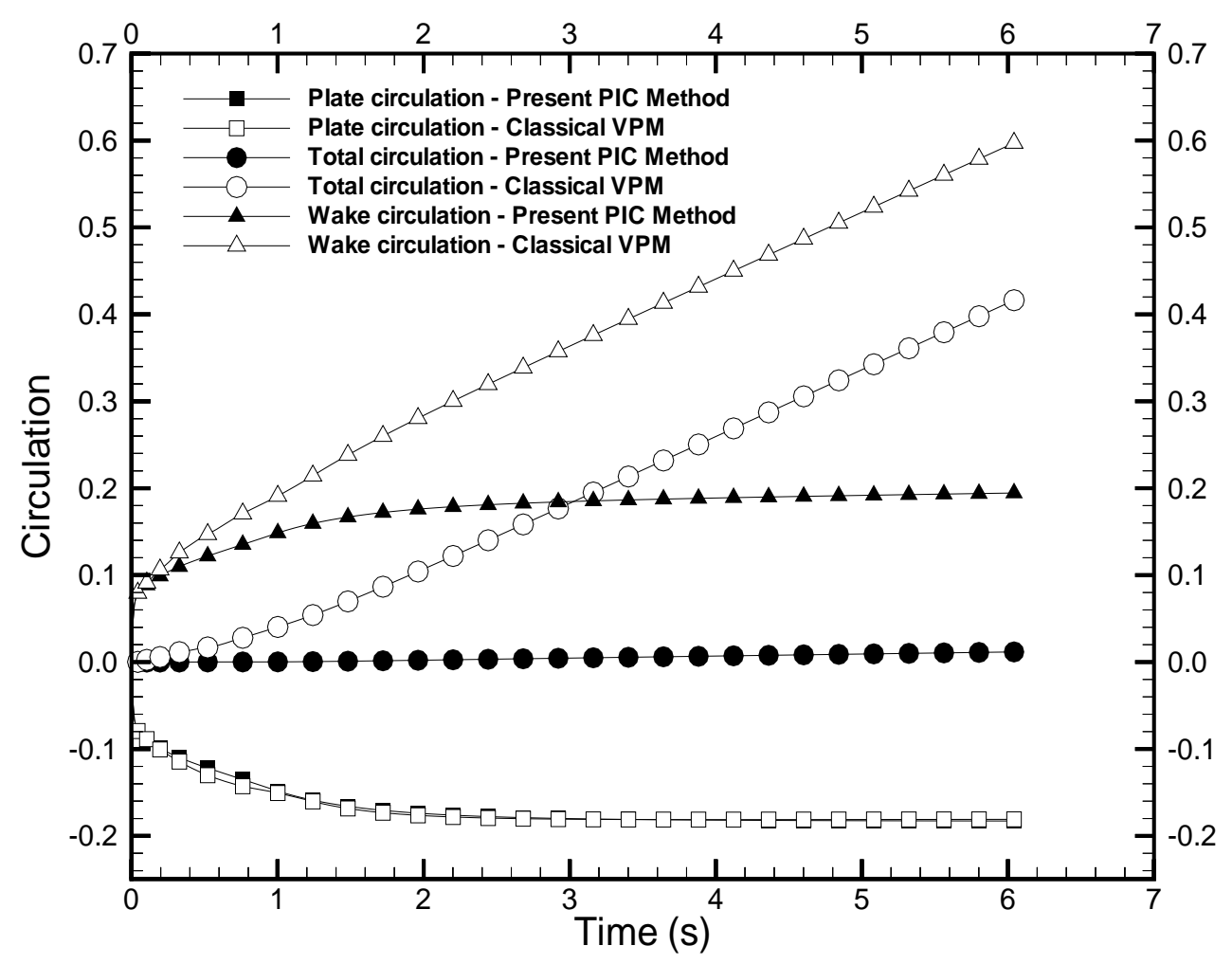

ESAIM : PROC., VOL. 7, 1999, 89-93 
Figure 6: Conservation of total circulation ( $\mathrm{AR}=1, \alpha=7.4$ deg., 50 panels).

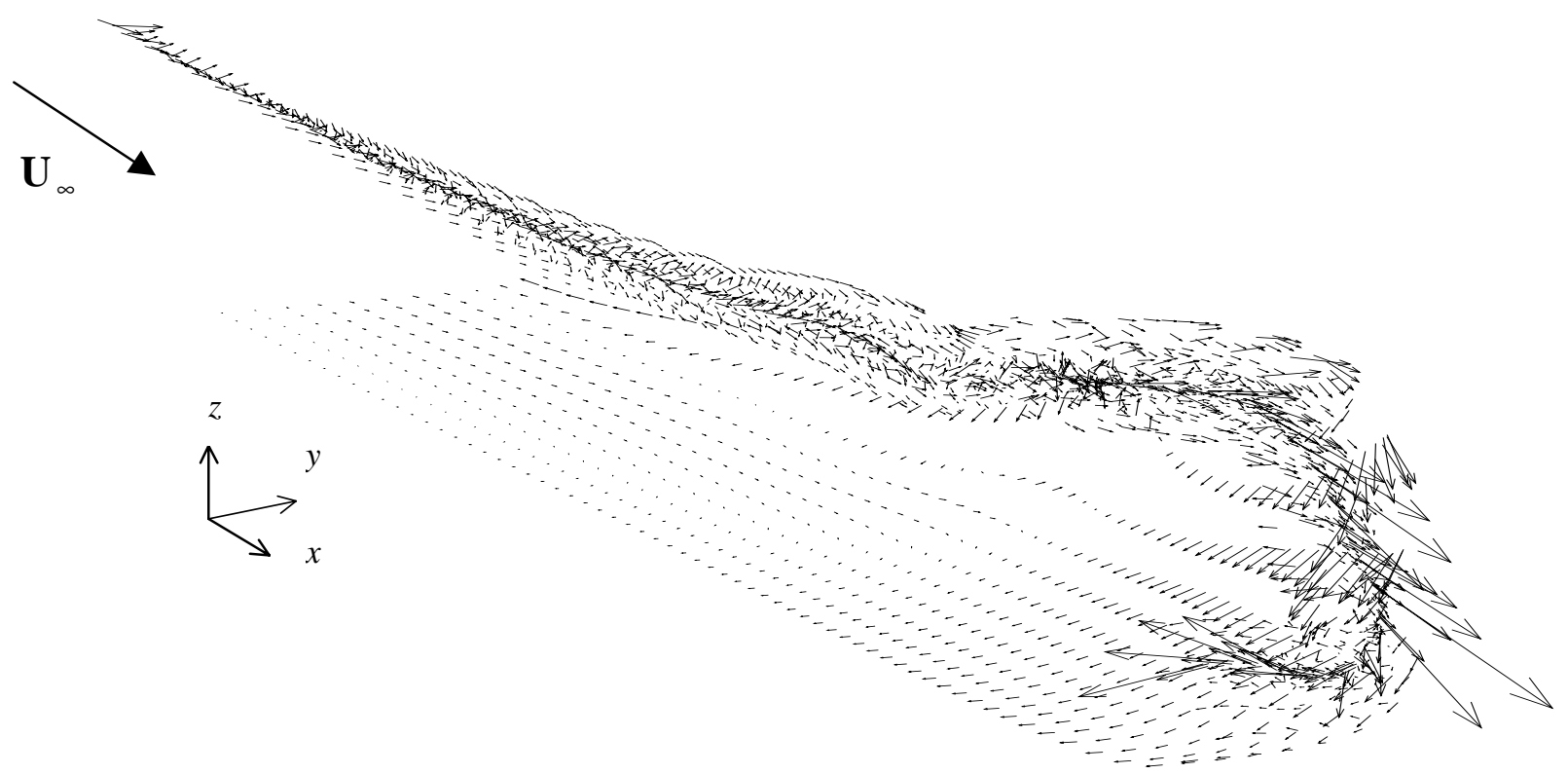

(a) Classical vortex particle method

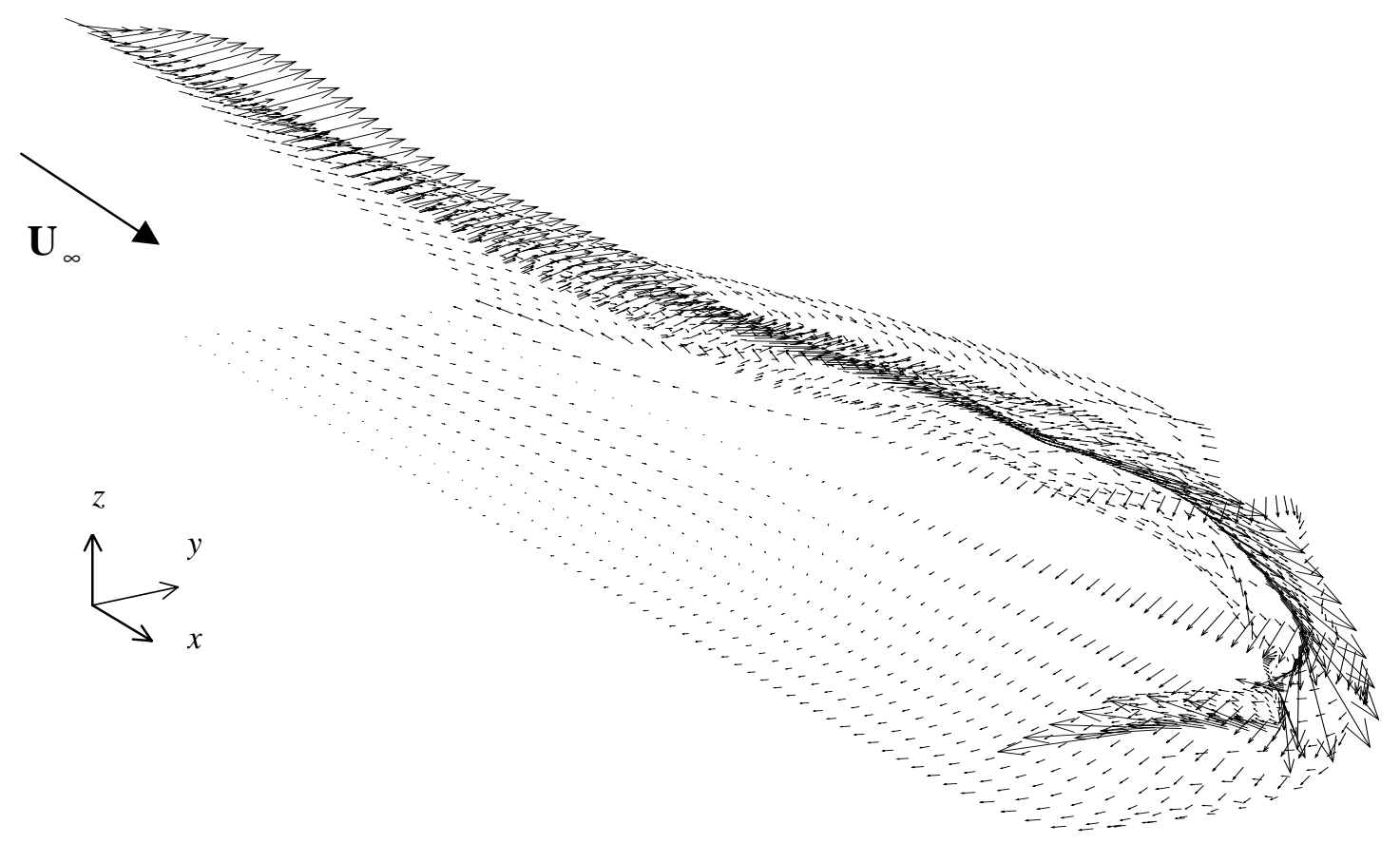

(b) Present PIC method

Figure 7: Particle vorticity field for $A R=1$ flat plate at $\alpha=11.3$ deg. ( $\left(t^{*}=2,200\right.$ panels, 2400 particles). Representation of the intensity vectors. 


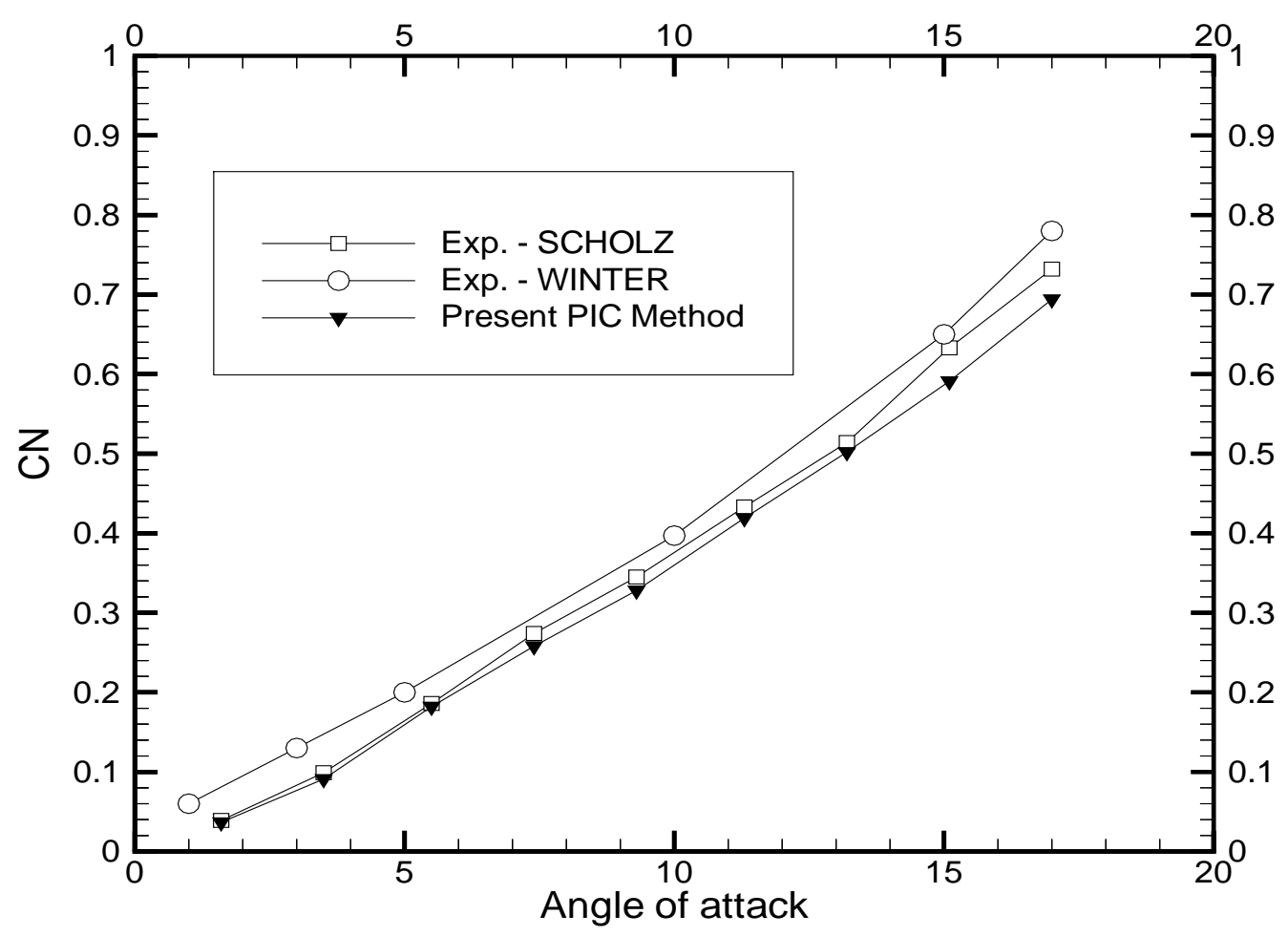

\begin{tabular}{|c|c|l|l|}
\hline $\begin{array}{c}\text { Angle of } \\
\text { attack } \\
\text { degrees) }\end{array}$ & $\begin{array}{c}\text { CN } \\
\text { Present PIC Method }\end{array}$ & $\begin{array}{c}\text { CN } \\
\text { Exp. Scholz }\end{array}$ & Error(\%) \\
\hline 1.6 & 0.036 & 0.039 & 7.6 \\
\hline 3.5 & 0.091 & 0.099 & 8.0 \\
\hline 5.5 & 0.182 & 0.186 & 2.1 \\
\hline 7.4 & 0.258 & 0.274 & 5.8 \\
\hline 9.3 & 0.328 & 0.345 & 4.9 \\
\hline 11.3 & 0.419 & 0.433 & 3.2 \\
\hline 13.2 & 0.502 & 0.514 & 2.3 \\
\hline 15.1 & 0.591 & 0.633 & 6.6 \\
\hline 17.0 & 0.694 & 0.732 & 5.2 \\
\hline
\end{tabular}

Figure 8: Normal force coefficients $\left(A R=1, t^{*}=5\right)$. Present method compared with experiment $[20,21]$. 


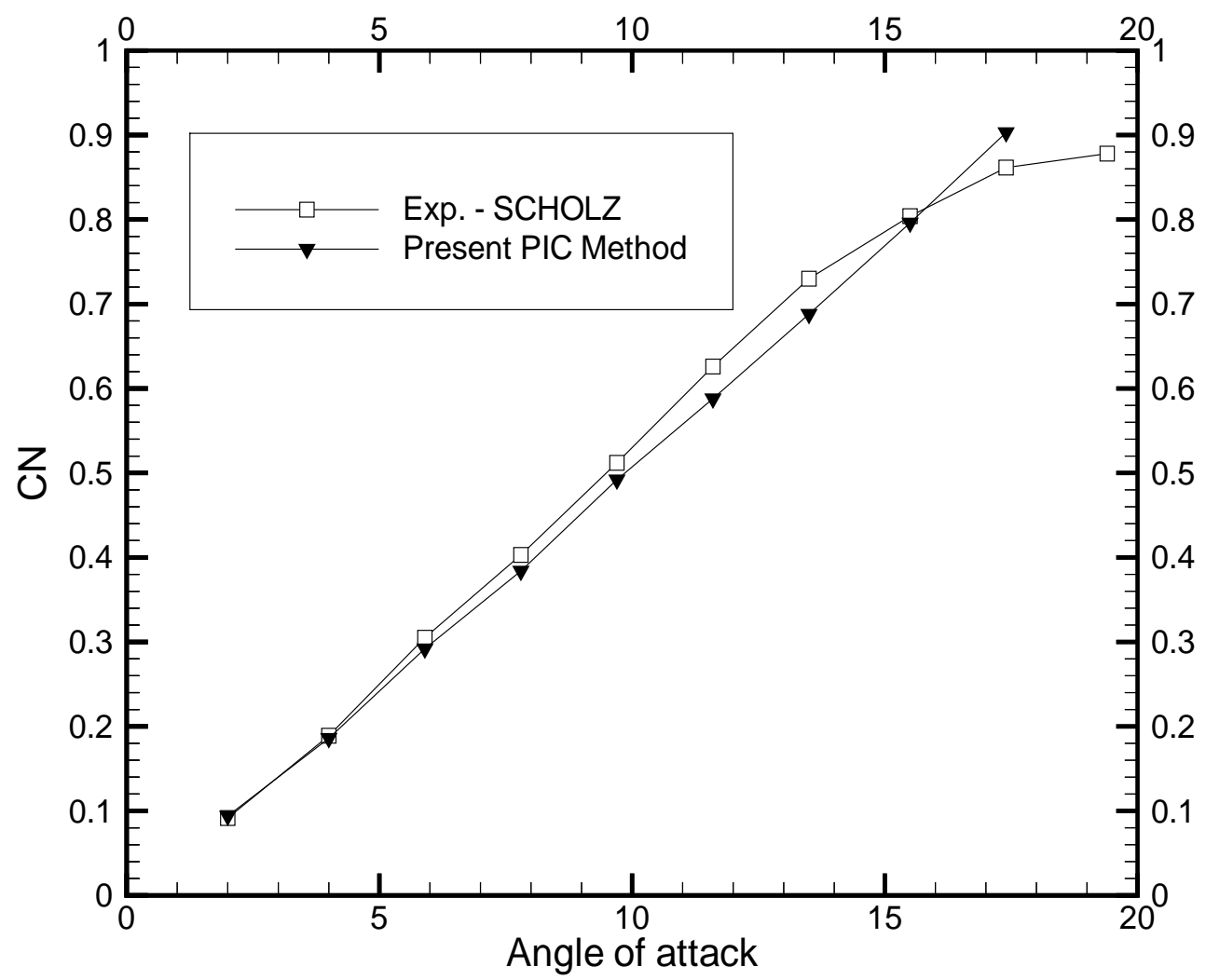

\begin{tabular}{|l|c|c|c|}
\hline $\begin{array}{c}\text { Angle of } \\
\text { attack } \\
\text { (degrees) }\end{array}$ & $\begin{array}{c}\text { CN } \\
\text { Present PIC Method }\end{array}$ & $\begin{array}{c}\text { CN } \\
\text { Exp. - Scholz }\end{array}$ & Error(\%) \\
\hline 2.0 & 0.094 & 0.0916 & 2.6 \\
\hline 4.0 & 0.186 & 0.189 & 1.6 \\
\hline 5.9 & 0.292 & 0.305 & 4.2 \\
\hline 7.8 & 0.384 & 0.403 & 4.7 \\
\hline 9.7 & 0.492 & 0.512 & 3.9 \\
\hline 11.6 & 0.588 & 0.626 & 6.0 \\
\hline 13.5 & 0.688 & 0.73 & 5.7 \\
\hline 15.5 & 0.796 & 0.804 & 1.0 \\
\hline 17.4 & 0.904 & 0.8615 & 4.9 \\
\hline
\end{tabular}

Figure 9: Normal force coefficients $\left(\mathrm{AR}=2, \mathrm{t}^{*}=5\right)$. Present method compared with experiment [20]. 


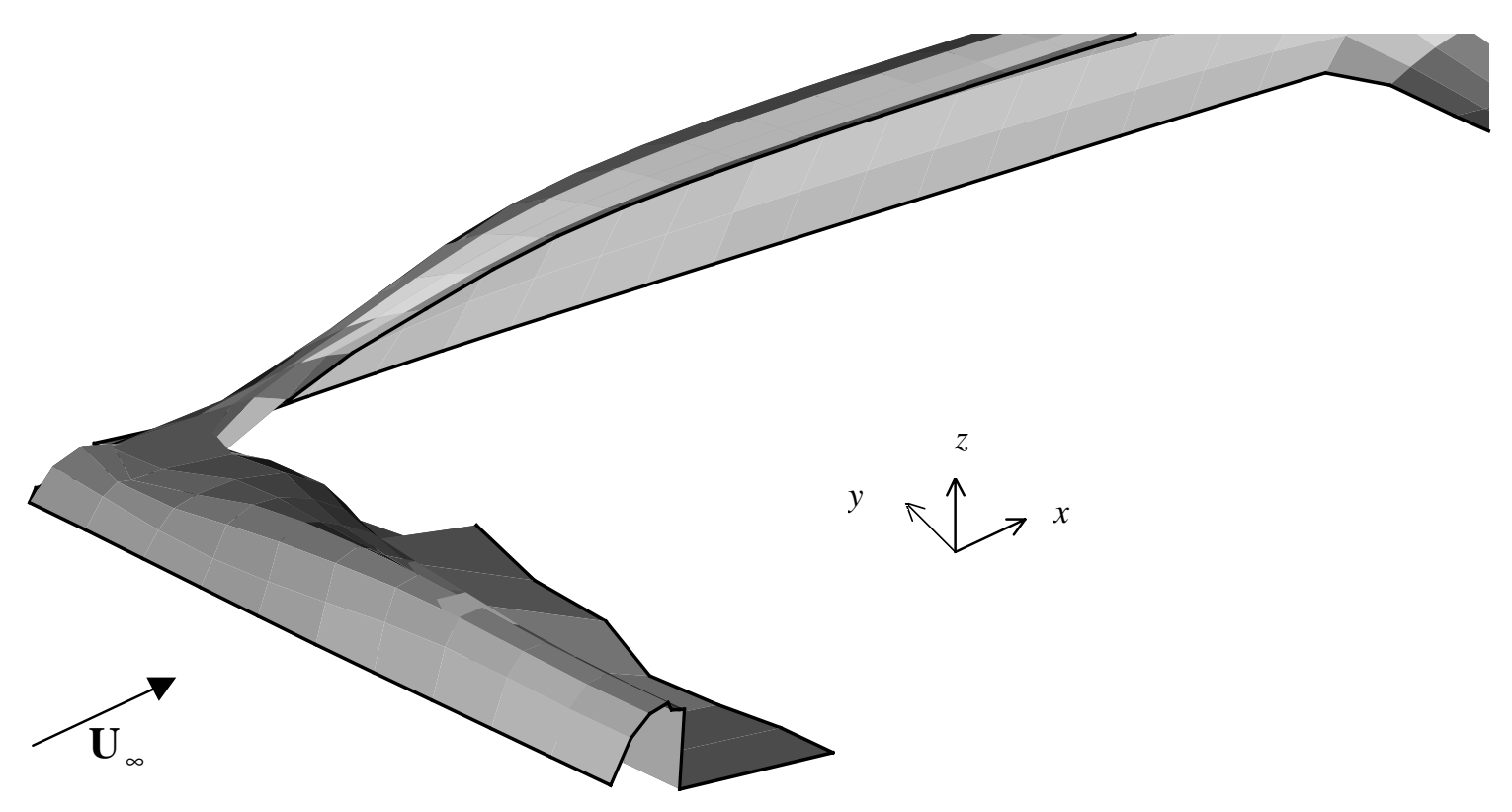

(a) Classical vortex particle method $\left(t^{*}=0.5,800\right.$ particles $)$

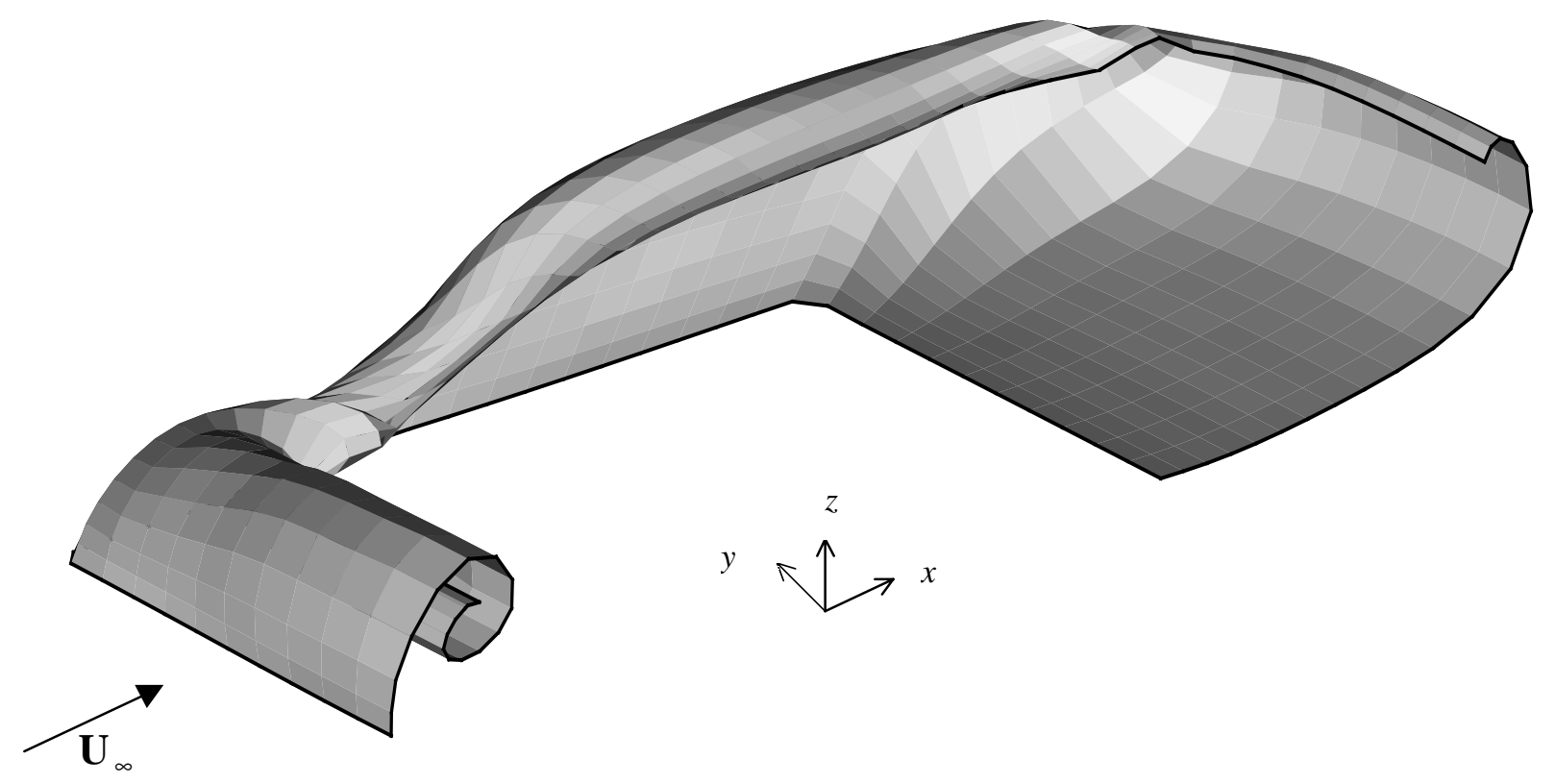

(b) Present PIC method ( $t^{*}=1,1600$ particles)

Figure 10: Separated flow around $A R=1$ flat plate at $\alpha=25$ deg. (200 panels). 